\title{
Optimal Path Planning using Hybrid Bat Algorithm and Cuckoo Search
}

\author{
Monica Sood $^{1 *}$, Dr. Sahil Verma ${ }^{2}$, Vinod Kumar Panchal ${ }^{3}$, Dr. Kavita ${ }^{4}$ \\ ${ }^{1,2}$ Department of Computer Science and Engineering, Lovely Professional University, Phagwara, India \\ ${ }^{3}$ Computational Intelligence Research Group (CiRG), Delhi, India \\ *Corresponding author:sahil.21915@lpu.co.in
}

\begin{abstract}
Path planning is key research topic in the field of robotics research, transportation, bioinformatics, virtual prototype desi gning, gaming, computer aided designs, and virtual reality estimation. In optimal path planning, it is important to determine the collision free optimal and shortest path. There may be various aspects to determine the optimal path based on workspace environment and obstacle types. In this research work, optimal path is determined based on the workspace environment having static obstacles and unknown environment area. A hybrid approach of meta-heuristic algorithm of Bat Algorithm (BA) and Cuckoo Search (CS) is used to determine the optimal path from defined source to destination. For experimentation, case study area of Alwar region, Rajasthan is considered which consist of urban and vegetation area. The reason for the selection of BA and CS for the path planning is the wide application and success of implementation of these concepts in the field of robotics and path planning. The consideration of individual BA for path planning can lead to problem of trapping between local optima. This obligates us to hybridize the concept of BA with some other efficient problem solving concept like CS. The hybridized concept of BA and CS is initially tested with standard benchmarks functions, after that considered for the application of path planning. Results of hybrid path planning concept are compared with individual CS and BA concepts in terms of simulation time and minimum number of iteration required to achieve the optimal path from defined source to destination. The evaluated results comparison of hybrid approach with individual concepts indicates the dominance of proposed hybrid concept in terms of standard benchmarks functions and other parameters as well.
\end{abstract}

Keywords: Optimal Path Planning; Meta-heuristic algorithm; Cuckoo Search; Bat Algorithm; Swarm Intelligence; Robotics

\section{Introduction}

Nature and natural elements have fascinated the human to design novel nature inspired algorithms which are efficient enough to solve the NP hard problems [1]. These nature inspired metaheuristic algorithms [2] include the Particle Swarm Optimization (PSO), Cuckoo Search (CS), Firefly Algorithm (FA), Ant Colony Optimization (ACO), Bat Algorithm (BA), and many more. Different algorithms are inspired from different natural elements like particle swarm optimization inspired from the flying birds and school of fishes, cuckoo search is inspired from the cleaver brood parasitic behavior of cuckoo to store the egg in other bird's nest, firefly algorithm is inspired from the flashing behavior of fireflies, ant colony optimization is inspired from the behavior of social species of ants, and bat algorithm is inspired from the echolocation behavior of bats. In this research paper, concepts of BA and CS are hybridized to solve the problem of path planning. Standard bat algorithm can trap in the local optima to solve any problem due to imbalance between the exploration and exploitation. So, individual BA cannot ensure the optimal path between the defined source and destination. On the other hand, CS has clever brood parasitic pattern mimicking property to store the egg in the nest of other bird. Moreover, CS is also efficient to solve path planning and other NP hard problems [3]. To further improve the performance of individual CS algorithm and resolve the problem of local optima in case of individual BA, hybrid concept of BA and CS is proposed. In this research work, hybrid proposed concept is ap- plied on the real time location of Alwar region, Rajasthan, India. This region consists of land cover features of dense vegetation region and urbanization area. Work space of static obstacles and unknown environment is used. Moreover, proposed hybrid concept is tested on standard benchmark functions to test the performance. The considered benchmark functions are Ackley Function, Shubert Function, Griewank Function, Rastrigin Function, Schwefel Function, De Jong Function, Easom Function, Michalewicz Function, and Rosenbrock Function. Based on the mentioned functions, proposed hybrid concept is tested and applied to solve the problem of optimal path planning. The results of path planning are evaluated in terms of simulation time and minimum number of iterations required to find the optimal path in the workspace with defined source and destination.

The organization of the other sections of the paper is as follows: Section 2 presents the work related to path planning using metaheuristic concepts. Section 3 brief about the basic concepts of cuckoo search and bat algorithm. Section 4 presents the proposed hybrid approach of BA \& CS. Section 5 performs the computational analysis of hybrid concept in terms of benchmark functions and other evaluated results in terms of minimum number of iterations \& simulation time. At last, Conclusion of the section is presented in Section 6. 


\section{Related work}

This section briefs the existing concepts for the path planning using meta-heuristic algorithms. There are various meta-heuristic concepts considered and modified by different authors to apply on the path planning. In this research paper, the work of latest and popular authors in the field of path planning is considered.

Wang et al. [4] introduced bat algorithm for path planning in unmanned combat air vehicles. Standard bat algorithm is modified with mutation property when the new solutions are updated and named as bat algorithm with mutation. A graph with nodes is considered and a global collision free optimal path is achieved by connecting the selected nodes. The nodes are selected on the basis of fitness function value. In this case, lower the fitness value, smaller the threat and higher the chances to select the node. The simulation results when compared to other meta-heuristic techniques, projects the viability of proposed algorithm.

Further, Mohanty and Parhi [5] proposed a cuckoo search based optimization concept for the robotic path planning in an unknown environment with multiple obstacles. The objective function of the cuckoo search is formulated to identify the best path without any collisions. The best solution results are obtained by cuckoo search and are sequentially visited by mobile robot. The robot keeps on moving on the particular path until an obstacle is detected. The comparative results of cuckoo search with genetic algorithm and PSO witnessed the effectiveness of cuckoo search for path planning. The authors further continued their work and proposed a new variant of cuckoo search algorithm for path planning problem in mobile robot navigation [6]. The information about the obstacles is obtained with the help of obstacles. The mobile robot moves sequentially on every path identified by cuckoo search algorithm in the form of best solution. The robot moves towards the destination until an obstacle is detected. The mobile robot uses the sensor information to detect the nearby obstacle and then cuckoo search is implemented to avoid collisions. Different scenarios are considered to compare the simulation results of cuckoo search with genetic algorithm and PSO and in every scenario cuckoo search outperforms all.

More recently, Das et al. [7] hybridized the improved PSO (IPSO) with differential perturbed velocity (DV) algorithm to identify multi-robot based optimal path in a clutter environment. The environment is known and obstacles are static in nature. But in case of multiple robots, one robot is considering other robot as a dynamic obstacle. In the proposed algorithm, robots work according to decentralized and centralized approach. In decentralized approach, each robot identifies an optimal collision free path based on individual memory information. In centralized approach, the objective function of all the robots is evaluated to identify the optimal path The velocities of all robots are managed according to the DV algorithm. The experiments are performed on khepera II miniature robot and are also compared with existing meta-heuristic algorithms. The obtained results work in agreement with the proposed algorithm. Another multi-robot path planning algorithm based on PSO is recently developed by Ayari and Bouamama [8]. The authors proposed a dynamic distributed PSO (D2PSO) algorithm to identify collision free optimal path in static and known environment. Each robot is considered as a particle and every particle attempts to find its collision free optimal path based on its own knowledge information. The global optimal path is identified by combining the information of all particles. The algorithm calculates two local optimal detectors: LODpbest and LODgbest, one for the particle best and other for the global best. The particles which are unable to provide both particle best and global best are replaced with restructured particles after a successive number of iterations. The evaluate results proved the applicability of D2PSO to path planning problem with multiple robots.

Englot and Hover [9] extended the popular traveling salesman problem of ant colony optimization to multi-goal path planning problem. The environment information is already known and
ACO is implemented to identify the optimal path from source to specified goal point. Since the problem consists of multiple goals, ACO is combined with sampling based probabilistic roadmap planner to detect the point-to-point feasible paths between the specific goals swiftly. ACO is considered to decide the order of the goals to be visited due to its meta-heuristic property and once ant moves on a new edge, rapid-exploring random tree (RRT) is implemented to detect the path. The algorithm is compared with the already existing sampling based multi-goal path planning algorithms and the experimental results work in agreement with the proposed algorithm.

Further, Wang et al. [10] modified the standard firefly algorithm for path planning in uninhabited combat air vehicle (UCAV). In the proposed algorithm fireflies communicate with top fireflies and exchange information with them while updating the light intensity information. For the random movement of the fireflies, another modification is the addition of levy flight property. In the proposed algorithm, the brightest fireflies are categorized as top fireflies. For each top firefly, another firefly of same category is randomly picked and the path connecting these two fireflies results into the generation of a new firefly. In case the two top fireflies have same intensity values then the new firefly is generated at the middle of the other two fireflies. The value of absorption coefficient is updated after each iteration. The modified firefly algorithm produced the best results with absorption coefficient value of 1.0

Recently, Das et al. [11] proposed a combination of PSO and gravitational search optimization (GSO) based algorithm for path planning of a multi-robot system in a clutter environment. In the given multi-robot system, each robot identifies its own path and makes decision. The robots are not only working individually but in a team as well. They communicate and coordinate with each other to identify the next position. The improved PSO-GSO algorithm aims to optimize the identified system path by minimizing its path length. The evaluation of the system is performed by comparing the results of improved PSO-GSO with individual improved PSO and improved GSO algorithms. The results indicate the betterment of improved PSO-GSO as compared to others.

\section{Basic concepts}

This section presents the basic fundaments and rules of considered concepts of Bat Algorithm and Cuckoo Search. These algorithms are used to propose the hybrid concept of BA and CS. The basic of $\mathrm{BA}$ and $\mathrm{CS}$ is presented here:

\subsection{Bat algorithm}

Bat Algorithm is nature inspired algorithm that work by imitating the echolocation behaviour of bats. Bat algorithm was proposed by Yang in 2010 [12][13]. Bat algorithm works based on the following rules:

1. Bats have the magical power to detect the distance with the help of echolocation and can easily differentiate between the food/prey and other objects of surroundings.

2. Bats use the variable of fixed frequency $f_{\min }$, loudness $A_{0}$, varying wavelength $\lambda$ to find the prey with a random fly with velocity $v_{t}$ from position $x_{t}$ Based on the target prey, bats can adjust the wavelength of emitted pulses and respective pulse rate emission $r$, where $r \in[0,1]$

3. Bats can adapt various means to vary loudness value but it is assumed that loudness can vary from minimum constant value $A_{\min }$ to maximum possible positive value $A_{0}$.

\subsection{Cuckoo search}

Cuckoo Search algorithm is also a nature inspired algorithm that work based on the brood parasitic nature of laying their eggs in 
other bird's nest. CS algorithm was proposed by Yang and Deb in 2009 [14][15]. CS is overall based on the following rules:

1. Cuckoo bird randomly choose a nest to dump its egg and single egg can be laid by each cuckoo bird once at a time.

2. The host nests that can provide quality solution (eggs) is considered for the next generations.

3. The count for the host nest is assumed to be fixed and the probability of host bird to recognize the alien egg is $p_{a} \in[0,1]$. If host bird recognized the alien egg, then host can either destroy the egg or can itself leave the nest to build a new nest for itself.

In CS, the timing of laying eggs and the selection of other bird's nest by some cuckoo bird species is astonishing. Cuckoo birds use the levy flight to generate nests at each iteration. The process of levy flight is performed by randomly selecting the position of egg. The values of objective function is calculated and compared. The selected egg is moved to new coordinates if the values are better than the previous values. This process is followed to find solution of any NP hard problem.

\section{Proposed hybrid approach}

This section presents the proposed hybrid concept of BA and CS to find the optimal path from source to destination. The main goal to use the meta-heuristic BA and CS is to obtain the optimized path with minimum possible iteration and simulation time. The proposed algorithm combines the properties of the two metaheuristic algorithms to handle the present obstacles and to find the optimal path from source point to destination point in a static and unknown environment. In this hybridized approach, BA uses the pattern mimicking property of cuckoo bird to handle the obstacle present and the concept of levy flight is used to randomly move the bats. In this research work, specific urbanization and dense vegetation regions are considered from the dataset of Alwar region, Rajasthan. The considered workspace region is considered as collection of binary pixels values: 0 and 1 where value 1 indicates obstacle free white pixel and value 0 is black pixel with obstacle. The step by step algorithm is discussed here:

\section{Algorithm}

Step 1: Consider the input classified Alwar image and define the source $s_{p}$ and destination $d_{p}$ for the path planning.

Step 2: Apply Morphological operation to reduce the blocked paths and unnecessary area gaps between the source to destination.

Step 3: Initialize the parameters of bat algorithm for n-bats possessing random position $x_{i}$ in d-dimensional search space. The other parameters used to move the bats are velocity $v_{i}$, minimum frequency $f_{\min }$, maximum frequency $f_{\max }$, constant pulse rate $r$, and loudness $A$.

Step 4: Evaluate the size $Q_{\text {bert }}$ and position $x_{\text {beat }}$ for the minimum fitness function value. The fitness function value can be evaluated using (1).

$f\left(x_{i}\right)=\sum_{k=\mathbb{1}}^{D+1} P_{k-1} P_{k}$

Where, $i=1,2, \ldots n, P_{k-1} P_{k}$ stands for distance between two adjacent nodes $P_{k-1}$ and $P_{k}$, and $n$ is the number of bats. The value of $P_{k}$ can be evaluated using (2).

$P_{i}=P_{i}^{(0)}+\left(P_{i}^{(1)} P_{i}^{(0)}\right) h_{i}$

Where, $h_{i}$ is the scale parameter that belongs to $[0,1]$ for $i=1,2$,

..., D. Here, $D$ stands for number of nodes, $P_{i}^{(0)}$ and $P_{i}^{[1]}$ are the endpoint of nodes.

Step 5: Further, evaluate the frequency $f_{i}$ and update the velocity $v_{0}^{t}$ and position $x_{i}^{t}$ using (3) to (5). $f_{i}=f_{\text {min }}+\beta\left(f_{\text {max }}-f_{\text {min }}\right)$

$v_{i}^{t}=v_{i}^{t-1}+\left(x_{t}^{t}-x^{c}\right) f_{i}$

$x_{i}^{t}=x_{i}^{t-1}+v_{i}^{t}$

Where, $\beta$ is uniform and random distribution factor lie between [0, $1], x^{*}$ is the globally best solution among the entire population. In this research work, $f_{\max }$ is considered as 100 and $f_{\min }$ is considered as 0 .

Step 6: If the value of random number is larger than the constant pulse rate $r$, then bats will follow the random walk process as evaluated with (6).

$x_{\text {พละ }}=x_{\text {Q }}+\in A^{t}$

Where, $A^{t}$ is the average loudness value of all the bats at any time $t$ and the value of random number $\epsilon$ lie between $[-1,1]$.

Step 7: The obstacles are handled with cuckoo search based property with an assumption of considering obstacle as worst nest for cuckoo egg. In this way, bats use the property of pattern mimicking to handle the obstacles.

Step 8: When the obstacle is detected then the Euclidian distance of the neighbour pixels from destination point is calculated to find the next point for the movement of bat agents. If $p_{\bar{f}}$ and dg; are pixel point and destination point respectively then the Euclidean distance between them can be evaluated by using (7).

$d_{i j}=\left\|p_{i}-d p_{j}\right\|=\sqrt{\sum_{k=1}^{M}\left(p_{i k}-d p_{j k}\right)^{2}}$

After, handling the obstacle, bats again proceed towards the destination by calculating the Euclidean distance from the neighboring pixels to destination point.

Step 9: Evaluate the bat algorithm based global optimum solution for all the pixel points in the way from source to destination using step 4 to step 8 .

Step 10: Obtain the final shortest optimum path from source to destination.

\section{Results and discussion}

This section presents the results of the proposed hybrid concept based on simulation time and minimum iteration required to obtain the shortest optimal path. The proposed hybrid concept is tested with benchmark functions before applying for the application of path planning. The experiment is performed on MATLAB version 8.3.0.532 and system configuration of window 7 operating system, 8 GB RAM, Intel i3 Processor. The used standard benchmark functions to test the proposed hybrid concept are Ackley Function, Shubert Function, Griewank Function, Rastrigin Function, Schwefel Function, De Jong Function, Easom Function, Michalewicz Function, and Rosenbrock Function. The results are evaluated with maximum possible repetition of hybrid approach to better analyze the results. The global optima results are evaluated until variation of results is observed lesser than $10^{-5}$. The evaluated values of these benchmarks functions using hybrid proposed algorithms along with values of individual BA and CS are illustrated in table 1 . In table 1 , values are expressed as average number of evaluations in success rate which is presented in the format of $x \pm y$, where $x$ is the mean of function evaluation value and $y$ is the standard deviation. 
Table 1: Comparison based on Standard Benchmark Functions

\begin{tabular}{|c|c|c|c|}
\hline $\begin{array}{l}\text { Benchmark Func- } \\
\text { tions/ } \\
\text { Algorithms } \\
\end{array}$ & $\begin{array}{l}\text { Cuckoo } \\
\text { Search }\end{array}$ & $\begin{array}{l}\text { Bat } \\
\text { Algorithm }\end{array}$ & $\begin{array}{l}\text { Hybrid BA } \\
\text { \& CS }\end{array}$ \\
\hline $\begin{array}{ll}\begin{array}{l}\text { Ackley } \\
(\mathrm{d}=128)\end{array} & \text { Function } \\
\end{array}$ & $4936 \pm 903$ & $6933 \pm 2317$ & $\begin{array}{l}4611 \\
1354\end{array}$ \\
\hline $\begin{array}{l}\text { Shubert Function (18 } \\
\text { minima) }\end{array}$ & $9770 \pm 3592$ & $11925 \pm 4049$ & $\begin{array}{l}9561 \\
3108 \\
\end{array}$ \\
\hline Griewank Function & $\begin{array}{ll}10912 & \pm \\
4050 & \end{array}$ & $9792 \pm 4732$ & $\begin{array}{l}9432 \\
3991\end{array}$ \\
\hline Rastrigin Function & $\begin{array}{ll}10354 \quad \pm \\
3755\end{array}$ & $12573 \pm 3372$ & $\begin{array}{l}9718 \\
3527\end{array}$ \\
\hline $\begin{array}{l}\text { Schwefel Function } \\
(\mathrm{d}=128)\end{array}$ & $8829 \pm 625$ & $8929 \pm 729$ & $7866 \pm 693$ \\
\hline $\begin{array}{l}\text { De Jong Function } \\
(\mathrm{d}=256)\end{array}$ & $4971 \pm 754$ & $5273 \pm 490$ & $4715 \pm 416$ \\
\hline Easom Function & $6751 \pm 1902$ & $7532 \pm 1702$ & $\begin{array}{l}6243 \\
2133 \\
\end{array}$ \\
\hline $\begin{array}{l}\text { Michalewicz Func- } \\
\text { tion }(d=16)\end{array}$ & $3221 \pm 519$ & $4752 \pm 753$ & $2856 \pm 632$ \\
\hline $\begin{array}{l}\text { Rosenbrock Function } \\
(\mathrm{d}=16)\end{array}$ & $5923 \pm 1937$ & $7923 \pm 3293$ & $\begin{array}{l}5189 \\
1458\end{array}$ \\
\hline
\end{tabular}

The evaluated path length obtained using the hybrid proposed concept is 246 pixels. The path length using individual approach of BA and CS are also same but the obtained optimal path length of 246 pixels using hybrid approach takes lesser simulation time and minimum number of iterations that are required to obtain the optimal path. The comparison of proposed hybrid concept based on the minimum number of iteration and simulation time is presented in table 2 .

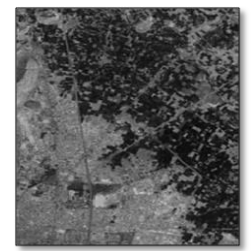

(a)

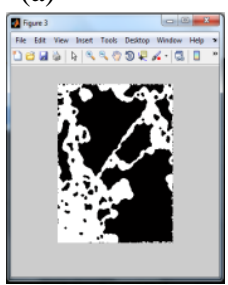

(c)

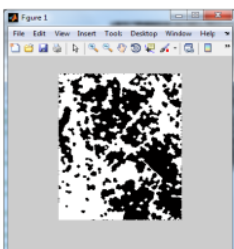

(b)

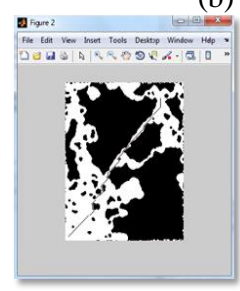

(d)
Fig. 1: (a) Red band satellite input image of Alwar region (b) the binary conversion of Satellite image (c) Image after morphological operation (d) Obtained Optimal Path Length

Table 2: Comparison of Hybrid proposed BA \& CS concept with Individual BA and CS

\begin{tabular}{|l|l|l|l|}
\hline & \multirow{2}{*}{$\begin{array}{l}\text { Minimum No. of Itera- } \\
\text { tions }\end{array}$} & Simulation Time (in sec) \\
\cline { 3 - 4 } & 49 & Minimum & Maximum \\
\hline CS & 61 & 179 & 230 \\
\hline BA & 32 & 154 & 264 \\
\hline $\begin{array}{l}\text { Hybrid BA \& } \\
\text { CS }\end{array}$ & & & 213 \\
\hline
\end{tabular}

From evaluated results values in terms of minimum number of iterations and simulation time, it can be noticed that proposed hybrid concept is efficient enough to find the optimal path length of 246 pixels. The minimum numbers of iterations consumed by hybrid approach are 32 whereas individual approaches need more iteration.

\section{Conclusion}

In this research work, hybrid approach of Bat Algorithm and Cuckoo Search is proposed to find the optimal path from defined source to destination. The proposed algorithm combines the properties of the two meta-heuristic algorithms to handle the present obstacles and to find the optimal path from source point to destination point in a static and unknown environment. In this hybridized approach, BA uses the pattern mimicking property of cuckoo bird to handle the obstacle present and the concept of levy flight is used to randomly move the bats. Proposed hybrid concept is also tested with standard benchmark functions before applying on path planning problem. The dataset of Alwar region situated in Rajasthan (India) is considered for the path planning. Results of path planning are tested in terms of minimum number of iterations and simulation time. From evaluated results, it can be declared that proposed hybrid concept is efficient enough to find the optimal path in comparison with individual BA and CS.

For future directions, CS can be hybridized with some other metaheuristic multi-agent based algorithm like firefly algorithm to further improve the minimum number of iteration and simulation time.

\section{References}

[1]. Yang, X. S. (2010). Nature-inspired metaheuristic algorithms. Luniver press.

[2]. Fister Jr, I., Yang, X. S., Fister, I., Brest, J., \& Fister, D. (2013). A brief review of nature-inspired algorithms for optimization. arXiv preprint arXiv: 1307.4186.

[3]. Mohanty, P. K., \& Parhi, D. R. (2016). Optimal path planning for a mobile robot using cuckoo search algorithm. Journal of Experimental \& Theoretical Artificial Intelligence, 28(1-2), 35-52.

[4]. Wang, G., Guo, L., Duan, H., Liu, L., \& Wang, H. (2012). A bat algorithm with mutation for UCAV path planning. The Scientific World Journal, 2012

[5]. Mohanty, P. K., \& Parhi, D. R. (2013, December). Cuckoo search algorithm for the mobile robot navigation. In International Conference on Swarm, Evolutionary, and Memetic Computing (pp. $527-$ 536). Springer, Cham.

[6]. Mohanty, P. K., \& Parhi, D. R. (2016). Optimal path planning for a mobile robot using cuckoo search algorithm. Journal of Experimental \& Theoretical Artificial Intelligence, 28(1-2), 35-52.

[7]. Das, P. K., Behera, H. S., Das, S., Tripathy, H. K., Panigrahi, B. K., \& Pradhan, S. K. (2016). A hybrid improved PSO-DV algorithm for multi-robot path planning in a clutter environment. Neurocomputing, 207, 735-753.

[8]. Ayari, A., \& Bouamama, S. (2017). A new multiple robot path planning algorithm: dynamic distributed particle swarm optimization. Robotics and Biomimetics, 4(1), 8.

[9]. Englot, B., \& Hover, F. (2011, May). Multi-goal feasible path planning using ant colony optimization. In Robotics and Automation (ICRA), 2011 IEEE International Conference on (pp. 2255-2260). IEEE.

[10]. Wang, G., Guo, L., Duan, H., Liu, L., \& Wang, H. (2012). A modified firefly algorithm for UCAV path planning. International Journal of Hybrid Information Technology, 5(3), 123-144.

[11]. Das, P. K., Behera, H. S., \& Panigrahi, B. K. (2016). A hybridization of an improved particle swarm optimization and gravitational search algorithm for multi-robot path planning. Swarm and Evolutionary Computation, 28, 14-28.

[12]. Yang, X. S. (2010). A new metaheuristic bat-inspired algorithm. In Nature inspired cooperative strategies for optimization (NICSO 2010) (pp. 65-74). Springer, Berlin, Heidelberg.

[13]. Yang, X. S., \& Hossein Gandomi, A. (2012). Bat algorithm: a novel approach for global engineering optimization. Engineering Computations, 29(5), 464-483.

[14]. Yang, X. S., \& Deb, S. (2009, December). Cuckoo search via Lévy flights. In Nature \& Biologically Inspired Computing, 2009. NaBIC 2009. World Congress on (pp. 210-214). IEEE.

[15]. Yang, X. S., \& Deb, S. (2010). Engineering optimisation by cuckoo search. International Journal of Mathematical Modelling and $\mathrm{Nu}$ merical Optimisation, 1(4), 330-343. 